\title{
Genome-wide identification, characterization and expression analysis of $B E S 1$ gene family in tomato
}

Deding $\mathrm{Su}^{1,2+}$, Wei Xiang ${ }^{1,2 \dagger}$, Ling Wen ${ }^{1,2}$, Wang Lu ${ }^{1,2}$, Yuan Shi ${ }^{1,2}$, Yudong Liu ${ }^{1,2^{*}}$ and Zhengguo $\mathrm{Li}^{1,2^{*}}$

\begin{abstract}
Background: As the key regulators in BR signaling, BES1 family genes regulate thousands of target genes involved in various development processes. So far, the functions of BES1 family are poorly understood in tomato, and a comprehensive genomic and expressional analysis is worth to conduct for this family.

Results: Here, nine SIBES1 family members were identified in tomato and classified into five groups based on the conserved motif, gene structure and phylogenetic analysis. Synteny among tomato, Arabidopsis, pepper and rice were further analyzed to obtain insights into evolutionary characteristics. Several cis-elements related to hormone, stress and plant development were exhibited in the promoter regions of SIBES1 family genes. Subcellular localization showed seven members localized both in the nucleus and cytoplasm, implying the presence of dephosphorylated and phosphorylated form of these seven proteins, furthermore, five of them possessed transcription activation activity whereas the left two functioned as transcriptional repressors. Another two members, however, neither localized in the nucleus nor had transactivation activity. Besides, SIBES1.8 showed flower-specific expression while other members expressed ubiquitously in all organs. Moreover, SIBES1 genes exhibited variational expression in response to nine principal plant hormones. Notably, the expression levels of SIBES1 genes presented a dominant downregulated trend in response to stresses.
\end{abstract}

Conclusions: In this study, we systematically analyzed the genomic characterization of SIBES1 family, together with the analyses of protein functional features and expression patterns, our results lay a foundation for the functional research of SIBES1 family.

Keywords: BES1 gene family, Genome-wide analysis, Subcellular localization, Transactivation activity, Expression pattern, Tomato (Solanum lycopersicum)

\section{Background}

Plant growth and development are continuously regulated by the integration of plant hormones. Meanwhile, their yield and quality are often influenced by various of environmental stimuli including biotic and abiotic stresses. To

\footnotetext{
*Correspondence: yudongliu@cqu.edu.cn; zhengguoli@cqu.edu.cn ${ }^{\dagger}$ Deding Su and Wei Xiang contributed equally to this work. 'Key Laboratory of Plant Hormones and Development Regulation of Chongqing, School of Life Sciences, Chongqing University, Chongqing 401331, China

Full list of author information is available at the end of the article
}

adapt the adverse environment, many genes especially for those transcription factors (TFs) tend to integrate multiple hormone signaling to against the environmental stimuli and maintain normal growth and development $[1,2]$.

It's well known that plant hormones contain but not limited to auxin, cytokinin (CK), gibberellin (GA), abscisic acid (ABA), ethylene (ET), brassinosteroid (BR), salicylic acid (SA), jasmonic acid (JA) and strigolactone. Among them, since the discovery in Brassica napus pollen, BR has been regarded as a plant growth-promoting hormone for its effects on cell elongation and division $[3,4]$.

(c) The Author(s). 2021 Open Access This article is licensed under a Creative Commons Attribution 4.0 International License, which permits use, sharing, adaptation, distribution and reproduction in any medium or format, as long as you give appropriate credit to the original author(s) and the source, provide a link to the Creative Commons licence, and indicate if changes were made. The images or other third party material in this article are included in the article's Creative Commons licence, unless indicated otherwise in a credit line to the material. If material is not included in the article's Creative Commons licence and your intended use is not permitted by statutory regulation or exceeds the permitted use, you will need to obtain permission directly from the copyright holder. To view a copy of this licence, visit http://creativecommons.org/licenses/by/4.0/. The Creative Commons Public Domain Dedication waiver (http://creativecommons.org/publicdomain/zero/1.0/) applies to the data made available in this article, unless otherwise stated in a credit line to the data. 
Subsequent studies indicate that BRs play multifunctional roles in plant developmental and physiological processes, including seed germination, plant architecture, vascular differentiation, stomata formation, flowering, male fertility, senescence, and stress resistance [5-7]. Meanwhile, the BR signal transduction pathway has been extensively explored by using genetic, molecular, and proteomic approaches over the past few decades, providing us a global view on the molecular mechanism of BR function. Briefly, an extracellular leucine-rich repeat receptor kinase (LRRRK), BRASSINOSTEROID-INSENSITIVE 1 (BRI1), can recognize and bind the $B R$, leading to the initiation of $B R$ signaling $[8,9]$. A series of kinases and phosphatases in the pathway are successively triggered to fulfil their functions, resulting the dephosphorylation and activation of two homologous transcription factors, BRI1-EMSSUPPRESSOR 1 (BES1) and BRASSINAZOLE-RESISTAN T 1 (BZR1) [10-13], which in turn regulate thousands of target genes by binding to the E-box (CANNTG) or BRresponse element (BRRE, CGTGT/CG) [14, 15].

The roles of BES1 and BZR1 in BR signal pathway are first illuminated by two dominant mutations, bes1-D and bzr1- $D$, which exhibit constitutive BR response phenotypes including suppressed bri1 dwarf phenotype, insensitivity to brassinazole (BRZ), elongated stem, accelerated senescence and upregulated expression of BR-induced genes $[12,13]$. Since then, the vitally important functions of BES1/BZR1 in integrating multiple hormone signaling to regulate plant growth and development are widely explored. For example, BES1/BZR1 can directly regulate the expression of CELLULOSE SYNTHASE GENES (CESAS) [16], MICROTUBULE DESTABILIZING PROTEIN 40 (MDP40) [17], ATBS1-INTERACTING FACTOR 2 (AIF2) [18], INCREASED LEAF INCLINATION 1 (ILI1), PACLOBUTRAZOL RESISTANCE 1 (PRE1) and ILI1 BINDING bHLH (IBH1) [19] or interact with MYB DOMAIN PROTEIN 30 (AtMYB30) [20], MYELOBLASTOSIS FAMILY TRANSCRIPTION FACTOR-LIKE 2 (MYBL2) [21] and HETEROTRIMERIC G-PROTEIN $\beta$ SUBUNIT (AGB1) [22] to control plant cell elongation. Analogously, BES1/ BZR1 can also influence plant immunity, stress responses, floral organ development and cell division and differentiation in quiescent center by directly regulating the expression of related key genes or interacting with relevant proteins [7]. A large number of putative target genes have been identified by chromatin immunoprecipitationmicroarray (ChIP-chip) studies for BES1 and BZR1, up to 1609 and 3410 respectively. Among which numerous genes are under the regulation of BRs, while there are a number of target genes involved in other signaling like plant hormone and stress signaling [23, 24], implying that BES1 and BZR1 are not only the master regulators in BR signaling but also play critical roles in other regulatory networks.
There are four BES1 homologs in A. thaliana, named $B E H 1-4$. However, few studies are focus on these four genes individually, probably because their functional redundancy in BR signaling [15]. In Arabidopsis, individual single mutant of BES1, BZR1 and their four homologs didn't show any growth defects. Moreover, no obvious phenotypes were observed from those different combinations of double, triple, and quadruple mutants. While the male sterility phenotype, tapetum and microsporocyte developmental defects, was exhibited in quintuple mutant (bes1 bzr1 beh1 beh3 beh4) and sextuple mutant (bes1 bzr1 beh1 beh2 beh3 beh4) [25]. Meanwhile, the similar results were demonstrated by another study reported by Chen et al. [26], indicating the highly functional redundancy of BES1 genes. The left two BES1 members, BAM7 and BAM8 (also named BMY4 and BMY2 correspondingly), are $\beta$-amylase proteins but included in BES1 family for the presence of BES1-type domain in the $\mathrm{N}$-terminal. Interestingly, these two $\beta$ amylases are reported to function as TF and function in controlling shoot growth and development by mediating BR signaling in Arabidopsis [27].

In a word, BES1 family genes act as key regulators in plant growth and development by orchestrating BR signaling and other signal pathways. However, understandings of BES1 gene family are mainly based on the studies performed in Arabidopsis, thus it's essential to obtain fresh insights from other plant species particularly from crops. As one of the most important horticultural crops, tomato (Solanum lycopersicum) is a typical model for the research of plant growth and development especially for the fleshyfruit development and ripening [28]. In our study, a comprehensive genome-wide analysis of SIBES1 gene family was performed, including their chromosomal distribution, conserved amino acid residues within the BES1-type domain, phylogenetic relationship, synteny analysis, gene structure, conserved motifs and potential cis-elements. We further explored their subcellular localization and transcriptional activation activity. What's more, the spatio-temporal expression patterns of SIBES1 gene family were also investigated. More important, we detailedly analyzed the responsiveness of SIBES1 gene family to the nine principal plant hormones and to different stresses. Our results provide valuable information to the functional and mechanism analysis of BES1 family genes in tomato. Moreover, this study may lay a foundation for the research of plant hormone signaling and stress resistance.

\section{Results}

Identification and characterization of SIBES1 gene family

To identify BES1 gene family in tomato, the conserved BES1-type domain sequence collected from AtBES1 was used as a BLASTP query in Solanaceae Genomics Database. Totally 9 putative SlBES1 candidates were obtained 
with default parameters. Meanwhile, Phytozome database was also used to search SIBES1 gene family, and the same SlBES1 candidates were obtained. Then the presence of conserved BES1-type domain was confirmed by CD-Search and SMART. These 9 SlBES1 genes was subsequently named as SlBES1.1 to SlBES1.9 according to their genomic locus (Table 1). Particularly, two members (SlBES1.1 and SlBES1.7) were annotated as $\beta$-amylases whereas other were annotated as TFs. The annotated seven SIBES1 TFs showed relative less exon number ranged from 2 to 3 , shorted protein length ranged from 180 to 333 amino acids (AA) and lighter predicted molecular weight ranged from 20,389 to $35,772.85 \mathrm{kDa}$. While those two annotated $\beta$-amylases had more exon number, longer protein length and bigger molecular weight (Table 1). Other detailed properties of SlBES1 genes like theoretical isoelectric point (pI) and BES1type domain position were also provided in Table 1.

\section{Chromosomal distribution and conserved amino acid residues analysis of SIBES1 genes}

SIBES1 gene family distributed on 8 chromosomes randomly, each SIBES1 gene located at one independent chromosome except chr.2 containing two SIBES1 genes, SlBES1.2 and SlBES1.3. Notably, most of SlBES1 genes positioned on distal ends of chromosomes, three of them distributed in a forward direction, while other six members distributed in a reverse direction (Fig. 1a).

The length of BES1-type domain was 86 to 135 amino acids in tomato. From the alignment of full length sequences, the comparative conserved sequences only showed in the N-terminal of BES1-type domain (Fig. 1b). We further analyzed the conservation of amino acids residues in this domain, similar to the analysis in A. thaliana, O. sativa and G. hirsutum [29], the amino acids residues in the $\mathrm{N}$-terminal BES1-type domain remained conserved at most of loci, which was assumed to be required for DNA binding. Remarkably, an arginine bias region between amino acids 8 to 13 was also observed in SlBES1 family. The C-terminal sequence of BES1-type domain was less conserved, it harbored many serine-rich phosphorylation sites in contrast, which implied the potentially regulatory center of SlBES1 proteins (Fig. 1c) [30].

\section{Phylogenetic and Syntenic analysis of SIBES1 genes}

To understand the phylogenetic relationship of SIBES1 family genes, a total of 59 BES1 genes from S. lycopersicum (9), A. thaliana (8), C. annuum (9), G. max (16), O. sativa (6) and $Z$. mays (11), were used to construct Neighbor-Joining phylogenetic tree by MEGA $X$ with default parameters. In keeping with the trees conducted by Liu et al. [29], Li et al. [31] and Song et al. [32], we grouped these 59 BES1 genes into five groups, named A to $E$, based on the bootstrap values and phylogenetic topology (Fig. 2a). Group A, B and E possessed the majority of BES1 genes and were further divided into 2 subgroups respectively. Subgroup A1 contained the key members $B E S 1$ and $B Z R 1$, which were the homologs of SlBES1.5 and SlBES1.9 respectively in tomato. As analyzed by Liu et al. [29], the corresponding group E was more ancient than other groups, and it was true that this group harbored BES1 genes from all of six species analyzed here. Additionally, genes in group $E$ showed quite longer amino acids length and were annotated as $\beta$ amylases discriminatively. Group D contained quite less $B E S 1$ genes from three species, including one tomato BES1 gene, SlBES1.8, and group C specifically possessed two BES1 genes from G. max, this result showed the expansion and divergence of BES1 gene family in evolution.

To further understand the phylogenetic mechanisms of SIBES1 family, a comparative syntenic maps was conducted among three dicots (tomato, pepper and Arabidopsis) and one monocot (rice) (Fig. 2b). The results showed that the most tomato BES1 homologs presented in pepper, another solanaceae species, followed by Arabidopsis, and the monocot rice exhibited the fewest homologs. What's more, all

Table 1 Characteristics of SIBES1 genes and the encoded proteins identified in tomato

\begin{tabular}{|c|c|c|c|c|c|c|c|}
\hline Gene name & Gene accession No. & Genomic locus & Exon number & $A A^{a}$ & $M W^{\mathbf{b}}(\mathrm{kDa})$ & $\mathrm{pl}^{\mathrm{c}}$ & BES1-type domains position \\
\hline SIBES1.1 & Solyc01g094580 & SL2.50ch01:85997496..86006359 & 11 & 695 & $77,864.45$ & 5.37 & 70-204 \\
\hline SIBES1.2 & Solyc02g063010 & SL2.50ch02:35030416..35032639 & 2 & 319 & $34,474.87$ & 9.38 & 38-119 \\
\hline SIBES1.3 & Solyc02g071990 & SL2.50ch02:41313401..41318179 & 3 & 324 & $34,908.89$ & 8.14 & $31-130$ \\
\hline SIBES1.4 & Solyc03g005990 & SL2.50ch03:667344..672399 & 3 & 323 & $34,696.65$ & 8.18 & $31-132$ \\
\hline SIBES1.5 & Solyc04g079980 & SL2.50ch04:64289859..64291884 & 2 & 328 & $35,108.38$ & 8.88 & 52-139 \\
\hline SIBES1.6 & Solyc07g062260 & SL2.50ch07:65038606..65041740 & 3 & 315 & $33,827.99$ & 9 & $3-99$ \\
\hline SIBES1.7 & Solyc08g005780 & SL2.50ch08:604998..612717 & 10 & 666 & $75,255.45$ & 6.09 & $72-202$ \\
\hline SIBES1.8 & Solyc10g076390 & SL2.50ch10:59363764..59364788 & 2 & 180 & 20,389 & 8.68 & $37-122$ \\
\hline SIBES1.9 & Solyc12g089040 & SL2.50ch12:64193208..64195373 & 2 & 333 & $35,772.85$ & 8.85 & 59-145 \\
\hline
\end{tabular}

${ }^{\mathrm{a}} \mathrm{AA}$ Number of amino acids; ${ }^{\mathrm{b}} \mathrm{MW}$ Molecular weight; ${ }^{\mathrm{c}} p$ / Theoretical Isoelectric point 


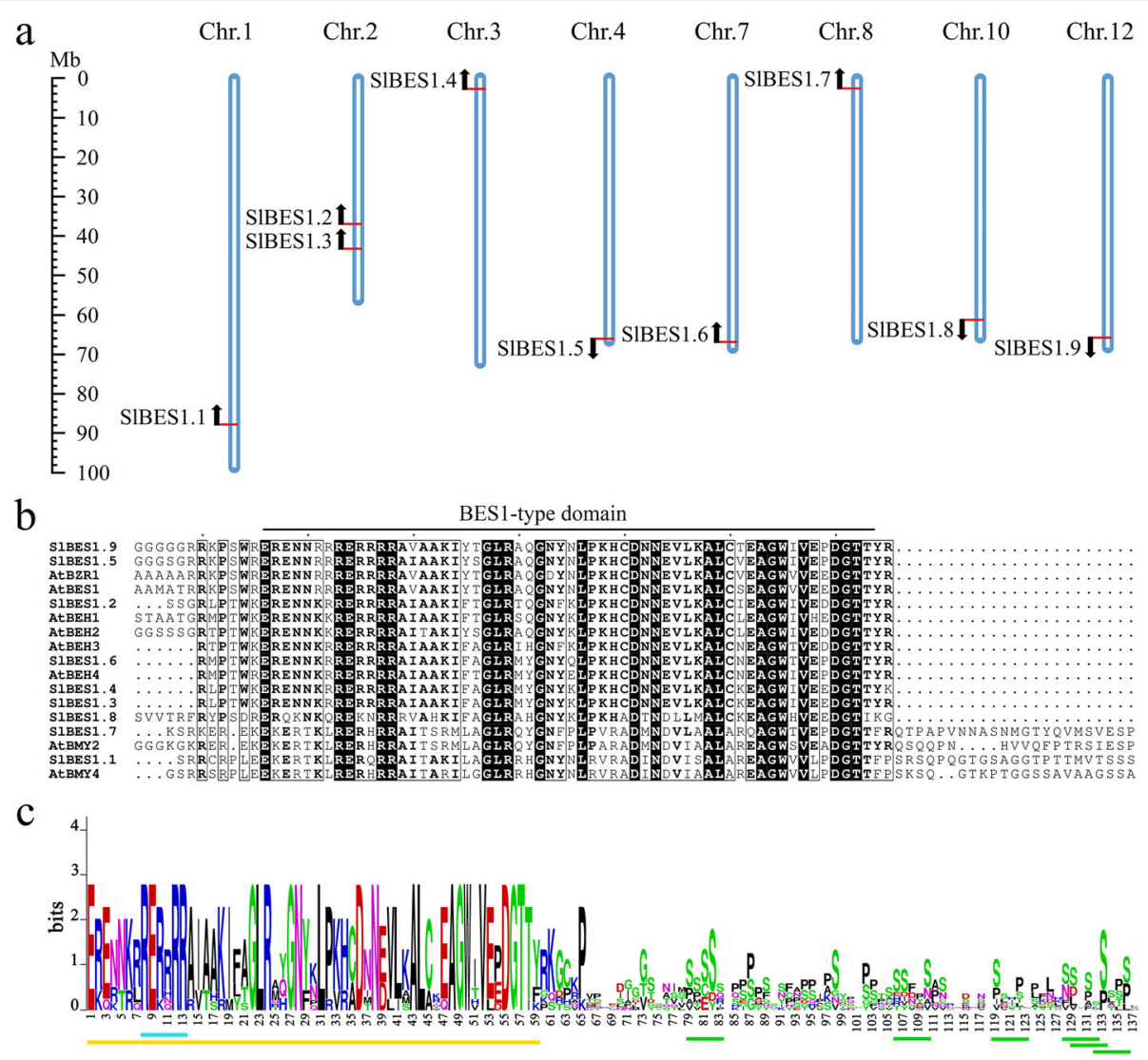

Fig. 1 Chromosomal distribution and conserved protein sequence analysis of SIBES1 genes. a Chromosomal distribution of the nine SIBES1 genes on tomato genome. Red line and arrow indicate the position and the direction of SIBES1 genes respectively. $\mathbf{b}$ Multiple sequence alignment of BES1-type domain (marked with underline) of tomato and Arabidopsis BES1 proteins by ClustalX. c Sequence logos of highly conserved amino acid residues of BES1-type domain in tomato. Yellow line indicates the conserved N-terminal BES1-type domain, blue line indicates the arginine composition biases, green lines indicate the serine-rich phosphorylation sites

SlBES1 syntenic genes (9) could be found on pepper chromosome, and most of SlBES1 syntenic genes (7) could be found on Arabidopsis chromosome, while only two exhibited on rice chromosome. Taken together, the syntenic gene pairs of SlBES1 were more presented in dicot than in monocot. Meanwhile, as the solanaceae relative of tomato, pepper possessed superior synteny with tomato than Arabidopsis and rice. These results suggested that BES1 family may play important roles to plant evolution.

\section{Gene structure and amino acids conserved motif of SIBES1 genes}

With the evolution, genes tend to diverge their regulatory and/or coding regions based on the gene duplication. Thus amino acid-altering substitutions and/or alterations may occur, and function of genes could be changed to adapt different growth conditions [33]. A simpler Neighbor-Joining phylogenetic tree was constructed by using BES1 protein sequences from $S$. lycopersicum and $A$. thaliana to fully analyze the gene structure and conserved motif (Fig. 3a).

Structures of BES1 genes clustered in the same clade were very close, including number and position of exons and introns. For example, the annotated $\beta$-amylase genes contained much more exons (10 to 11 ) than those annotated TFs that obtained only 2 to 3 exons generally, and the third exon of those three tomato BES1 genes (SIBES1.3, SlBES1.4 and SIBES1.6) had only 4 nucleotides. Furthermore, most of introns of tomato BES1 genes appeared to be longer than their Arabidopsis homologs, which agreed with the fact that tomato had the bigger genome. Besides, the BES1-type domain of tomato BES1 genes was all located between exon1 and exon2 except SIBES1.1. Noticeably, a LxLxL type ethylene-responsive element binding factor-associated amphiphilic repression (EAR) motif, which was previously reported as a negative transcriptional regulatory motif [34], was observed in the C-terminal end of those BES1 genes annotated as TFs, implying a potential transcriptional inhibition function of 


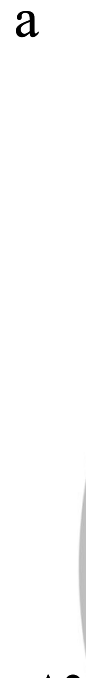

b
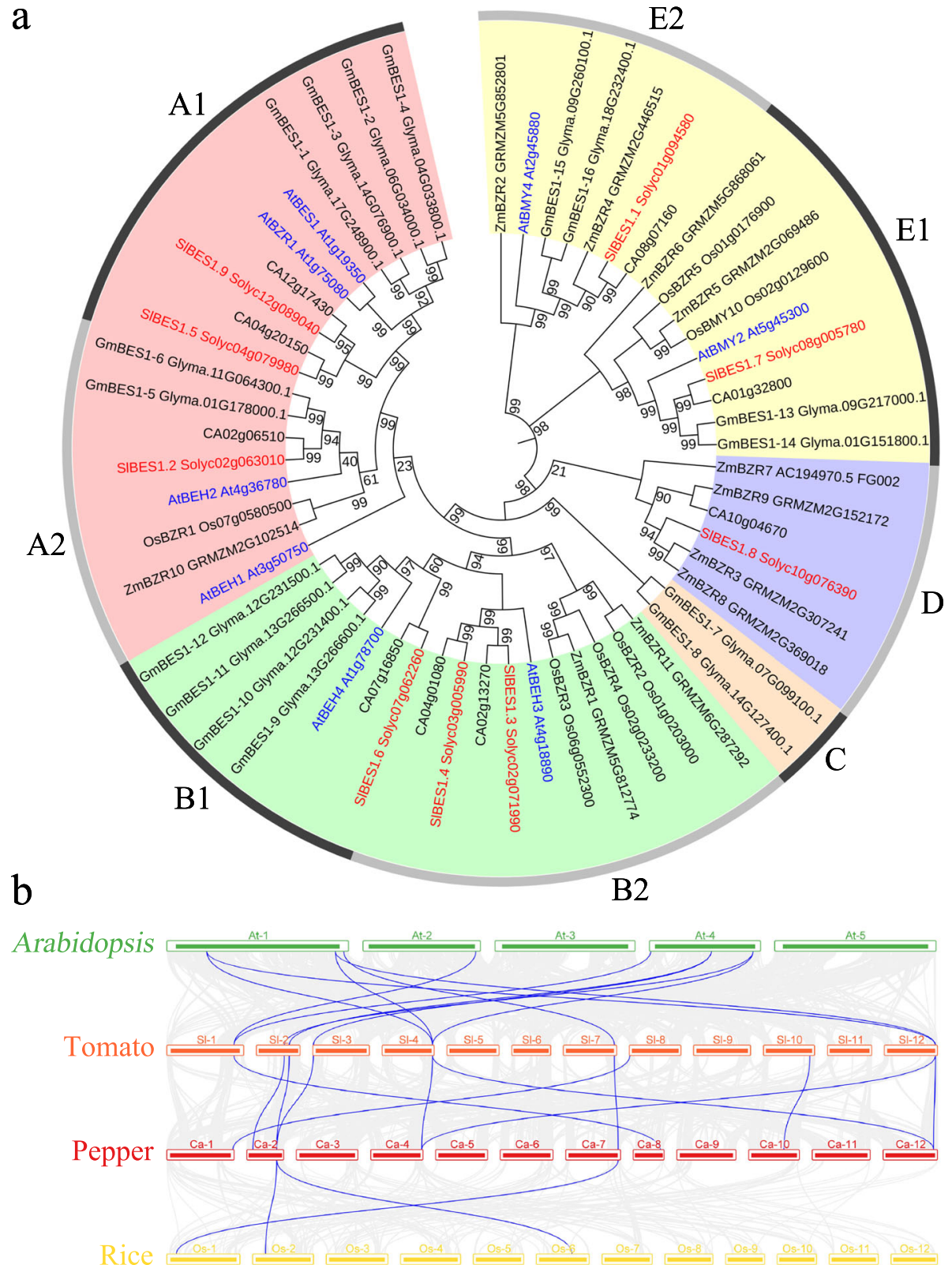

Fig. 2 Phylogenetic analysis of SIBES1 family. a Phylogenetic tree of BES1 proteins from tomato and other plants. The phylogenetic tree was constructed by MEGA X according to NJ method. 9, 8, 9, 16, 6 and 11 BES1 protein sequences from S. lycopersicum (red font), A. thaliana (blue font), C. annuum, G. max, O. sativa and Z. mays respectively were used. Group A-E are distinguished by different colors. Bootstrap values are provided near nodes. $\mathbf{b}$ Synteny analysis of SIBES1 genes among tomato, pepper, Arabidopsis and rice. The gray lines indicated the collinear blocks within these four species genomes, and the syntenic BES1 gene pairs were highlighted with the blue lines

these BES1 genes, while those annotated $\beta$-amylase genes didn't contain this special motif (Fig. 3b).

Proteins containing highly consistent amino acid sequences particularly in functional domain tended to share similar biological functions, thus 10 conserved motifs of tomato and Arabidopsis BES1 proteins were explored by the MEME suite (Fig. S1). As shown in Fig. 3c, motif 1 was the most conserved motif exhibited in all BES1 proteins and it overlapped with BES1-type domain.
The permutation and combination of these motifs were very closely related with their phylogenetic relationship. For example, group A and B shared the same motifs (motif 1, 5, 6, 7, 8 and 10) while exhibited an opposite order between motif 8 and 10, and the rest of motifs (motif 1, 2, 3, 4 and 9) were included into group E. Specially, group D exclusively contained the motif 1 , suggesting a potential loss of function or functional differentiation of gene in this group. 


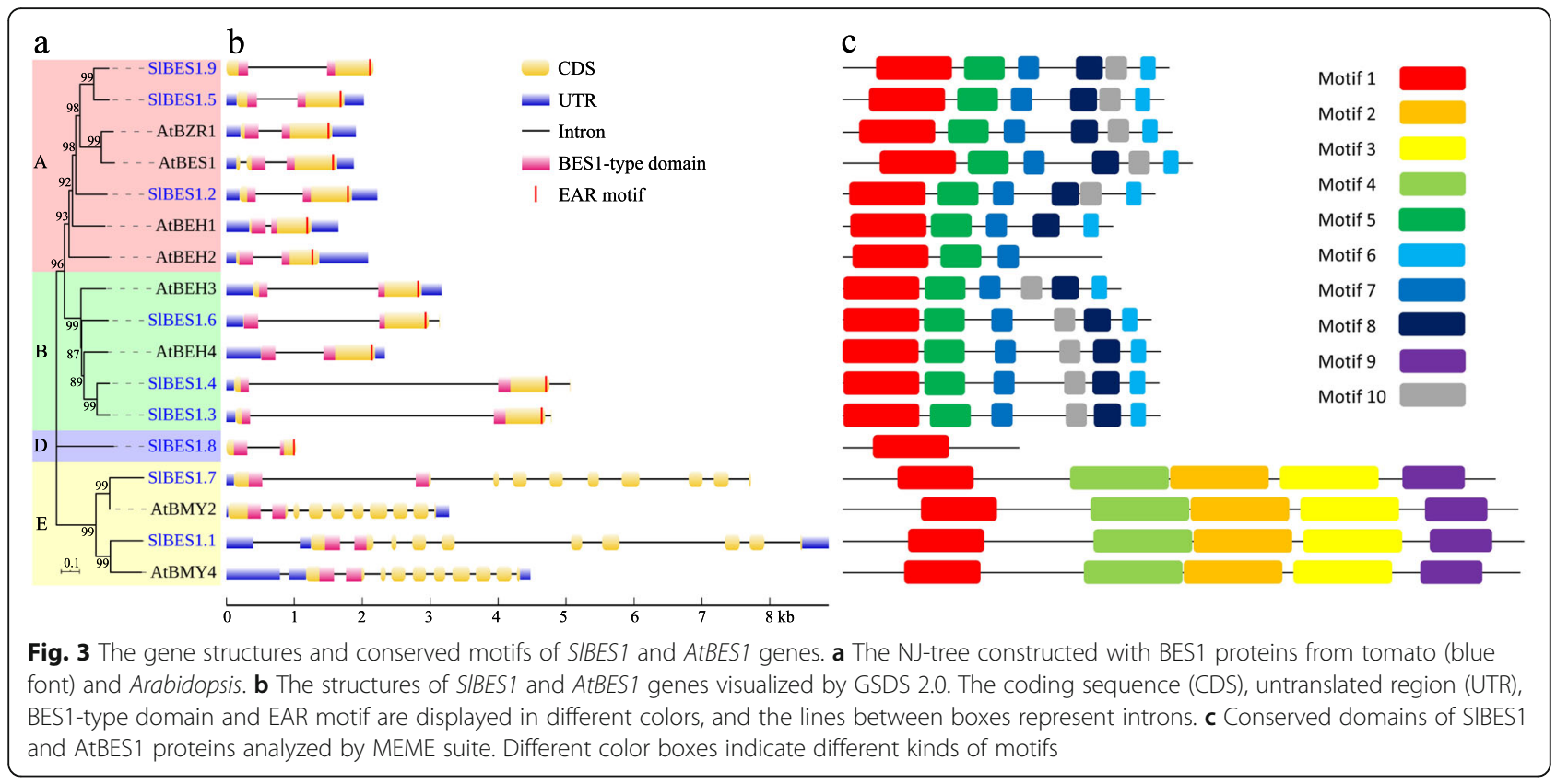

\section{Potential cis-element in SIBES1 gene promoters}

To explore the potential cis-elements, $2 \mathrm{~kb}$ upstream sequence of SlBES1 genes were submitted to PlantCARE database. The kind and position of cis-elements were marked as different icons (Fig. 4a), and their potential functions were annotated in Fig. 4b. All of these cis-elements detected could be mainly classified into three types: phytohormone responsive, plant development-related and stress responsive elements.
Among these cis-elements, ABRE and STRE were conspicuous, which were involved in abscisic acid and stress responsiveness respectively, indicating that SIBES1 genes may be able to be induced or repressed by abiotic stress and subsequently participate in plant stress resistance. Besides, each of SlBES1 gene possessed different kinds and amount of cis-elements, we may assume that, under different growing status and environmental conditions, SlBES1 genes could

\begin{tabular}{|c|c|c|c|}
\hline a & \multicolumn{3}{|l|}{ b } \\
\hline SIBES1.1 $=0$ & Element name & Symbol & Function annotation \\
\hline & ABRE & 0 & cis-acting element involved in the abscisic acid responsiveness \\
\hline $\mathrm{ES} 1.2 \Longrightarrow$ & ARE & $\Delta$ & cis-acting regulatory element essential for the anaerobic induction \\
\hline SIBES1.3 & AuxRR-core & 1 & cis-acting regulatory element involved in auxin responsiveness \\
\hline & Box III & $\mathrm{I}$ & protein binding site \\
\hline SIBES1.4 & CAT-box & 】 & cis-acting regulatory element related to meristem expression \\
\hline & CGTCA-motif & $\nabla$ & cis-acting regulatory element involved in the MeJA-responsiveness \\
\hline SIBES1.5 $=$ & DRE1 & II & Drought-responsive cis-regulatory elements \\
\hline SIBES1.6 th $=\mathrm{t}=\mathrm{t}=\mathrm{0}$ & GCN4_motif & $\theta$ & cis-regulatory element involved in endosperm expression \\
\hline 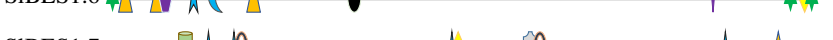 & HD-Zip 1 & $\theta$ & element involved in differentiation of the palisade mesophyll cells \\
\hline 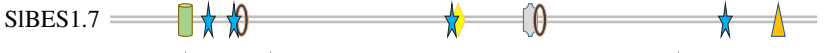 & $\begin{array}{l}\text { LTR } \\
\text { MBS }\end{array}$ & 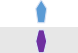 & $\begin{array}{l}\text { cis-acting element involved in low-temperature responsiveness } \\
\text { MYB binding site involved in drought-inducibility }\end{array}$ \\
\hline SIBES1.8=0th & MBSI & $\theta$ & MYB binding site involved in flavonoid biosynthetic genes regulation \\
\hline SIPES1 9 & O2-site & t & cis-acting regulatory element involved in zein metabolism regulation \\
\hline SIBES1.9 $=$ & P-box & 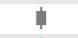 & gibberellin-responsive element \\
\hline$-2000 \mathrm{bp}$ & STRE & * & Stress response element \\
\hline & TATC-box & 0 & cis-acting element involved in gibberellin-responsiveness \\
\hline ABRE $\$ ARE $\triangle$ AuxRR-core $\|$ Box III $\$ CAT-box & $\begin{array}{l}\text { TC-rich repeats } \\
\text { TCA-element }\end{array}$ & 0 & $\begin{array}{l}\text { cis-acting element tinvolved in defense and stress responsiveness } \\
\text { cis-acting element involved in salicylic acid responsiveness }\end{array}$ \\
\hline GCN4_motif $\bigotimes$ HD-Zip $1 \theta$ LTR $\$ MBS $\$ MBSI $\theta$ O2-site $\nmid$ P-box & TGA-box & $\boldsymbol{v}$ & part of an auxin-responsive element \\
\hline TOTC G & TGA-element & ( & auxin-responsive element \\
\hline TATC-box [] TC-rich repeats $]$ TCA-element 0 TGA-box TGA-element ( WUN-motif & WUN-motif & $\frac{1}{k}$ & Wound-responsive element \\
\hline
\end{tabular}


function independently or synergistically to ensure plant normal growth and development.

\section{Subcellular localization of SIBES1 proteins}

Subcellular localization implied the working position of a protein and was nonnegligible for gene functional research. To detect the subcellular localization of SlBES1 proteins, green fluorescent protein (GFP) fused with SIBES1 proteins was used to transiently express in tobacco (Nicotiana benthamiana) leaf. As shown in Fig. 5, seven BES1 proteins, SlBES1.2, SIBES1.3, SlBES1.4, SIBES1.5, SIBES1.6, SIBES1.8 and SIBES1.9, localized both in the nucleus and cytoplasm. This result was basically consistent with the fact that phosphorylated BES1 mainly distributed in the cytoplasm while dephosphorylated BES1 accumulated in the nucleus [15]. For those two annotated $\beta$-amylase proteins, SlBES1.1 and SIBES1.7, the green fluorescence pigment showed a nonnuclear shape, thus we further used DAPI to mark the nucleus of tobacco leaf cell, and the green fluorescence pigment was truly not overlapped with the nucleus (Fig. S2). The chlorophyll auto-fluorescence was also detected to analyze if these two proteins localized to the chloroplast, while clear distinction was observed between these two fluorescence pigments both in the size and position, indicating a non-chloroplastic localization (Fig. S2). Taken together, given the bigger size of the green fluorescence pigment than the nucleus, we assumed that these two annotated $\beta$-amylase genes localized in the endoplasmic reticulum.

\section{Transactivation activity analysis of SIBES1 proteins}

As most of SlBES1 genes were annotated as TFs, the transcriptional activation activity was necessary to be analyzed, hence the GAL4-responsive reporter system in yeast was used to detect the transactivation activity of SlBES1 proteins (Fig. 6a). After transformed the pGBKT7-SIBES1 fusion plasmids into yeast for 3 days, all yeast transformants grew well on SD/-Trp medium, while only those five yeast transformants containing pGBKT7-SlBES1.3, pGBKT7SIBES1.4, pGBKT7-SIBES1.5, pGBKT7-SIBES1.6, pGBKT7SIBES1.9 respectively and positive yeast transformant hydrolyzed X- $\alpha-G a l$ and showed the blue pigment and survived from Aureobasidin A (AbA) screening, indicating that these five SlBES1s had transactivation activity whereas other four SlBES1s, including SlBES1.1, SlBES1.2, SlBES1.7 and SlBES1.8, had no transactivation activity. According to the non-nuclear subcellular localization of SIBES1.1 and SIBES1.7 presented above, we assumed that these two SIBES1 proteins were not TFs (Fig. 5), consistent with this assumption, SIBES1.1 and SIBES1.7 truly didn't have the transactivation activity (Fig. 6a). However, given the presence of EAR motif in the C-terminal end of those seven SIBES1 TFs (Fig. 3b), it was unexpected that five of them discovered to possess the transactivation activity (further discussed in Discussion). The rest of two SIBES1 proteins, SIBES1.2 and SIBES1.8, showing had no transactivation activity, were further to be ascertained if they acted as transcriptional repressor by dual-luciferase assay (Fig. 6b). Full length of coding sequences of these two genes were fused with GAL4 DNA binding domain as the effector. A strong transcriptional activator, VP16 [35], was used as a positive control. After coexpressed of effector and reporter in tobacco leaf, the LUC and REN value was measured. As anticipated, the relative LUC/REN ratios of pBD-SIBES1.2 and pBD-SIBES1.8 were pretty lower than the $\mathrm{pBD}$ alone. As a contrast, the VP16 transcriptional activator significantly increased the expression of the LUC reporter. Together with the transactivation activity analysis in yeast, we confirmed that SIBES1.2 and SIBES1.8 acted as the transcriptional repressor.

\section{Tissue-specific and spatio-temporal expression profiles of SIBES1 genes}

Development- and tissue-specific expression pattern could lead us to predict the potential function of a gene, thus the spatio-temporal expressions of SlBES1 genes were explored by quantitative real-time

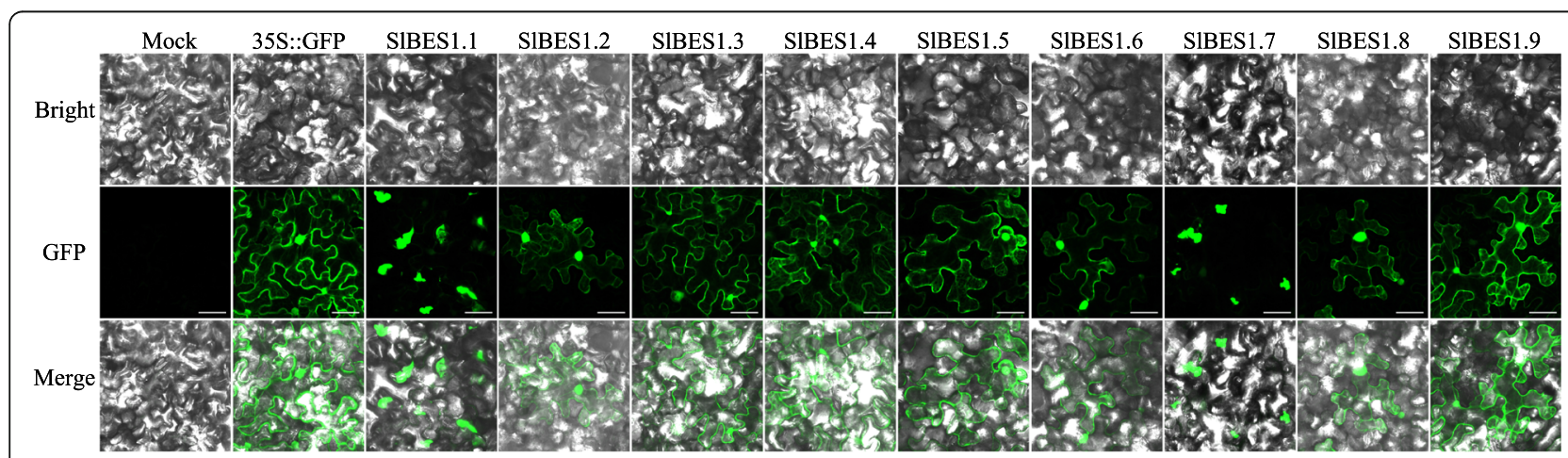

Fig. 5 Subcellular localization analysis of SIBES1 proteins. Tobacco (Nicotiana benthamiana) leaves transiently expressed SIBES1-GFP fusion proteins were observed through the laser scanning confocal microscope. Scale bars represent $50 \mu \mathrm{m}$ 


$\begin{aligned} & \text { Negative } \\ & \text { Positive }\end{aligned}$
SIBES1.1
SIBES1.2
SIBES1.3
SIBES1.4
SIBES1.5
SIBES1.6

polymerase chain reaction (qRT-PCR). Twenty two templates of tomato tissue were selected for expression profile detection, including seedling at 12 days post germination (DPG), root, stem, leaf at $30 \mathrm{DPG}$, flower and floral organ (sepal, petal, stamen and ovary) at anthesis and 2 days before anthesis and fruit at different development stages (7 days and 15 days after anthesis, immature green, mature green, breaker, 2 days, 4 days and 7 days after breaker). In general, most of SlBES1 genes expressed ubiquitously in all organs detected except SlBES1.8 that principally expressed in flower organ, indicating a potential important function of SlBES1.8 during fruit set. Notably, SIBES1.1 and SIBES1.4 had the relative stable expression pattern, only a relative higher expression was observed in anthesis stamen and petal respectively, suggesting that SlBES1.1 and SlBES1.4 may function fundamentally to tomato plant development. What's more, the expressions of SIBES1.2, SIBES1.5, SIBES1.6 and SIBES1.9 gradually increased with the development of fruit, reaching the highest level at IMG and MG stages, then decreased gradually with the fruit ripening (Fig. 7). Interestingly, these four genes possessed more close evolutionary relationship than other SlBES1 members (Fig. 3a), implying a potential functional redundancy or synergistic effect of these four SlBES1 genes to tomato fruit development.

\section{Expression profiles of SIBES1 genes in response to plant hormone}

It has been widely studied in the past century that plant hormones played vitally important roles in the regulation of plant growth and development. Understanding of the responsiveness of a gene to plant hormone especially for those TFs could provide us the clue in the research of gene function. In this study, nine major kinds of plant hormones or their analogues, including indole-3-acetic acid (IAA), 6Benzylaminopurine (6-BA), Gibberellin A3 $\left(\mathrm{GA}_{3}\right)$, Abscisic Acid (ABA), ethephon, epi-brassinolide (EBL), salicylic acid (SA), methyl jasmonate (MeJA) and strigolactone (GR24), were used to treat the tomato seedling at 12 DPG.

First of all, the efficient effects of plant hormone treatment were validated by the reference genes that were reported previously had responsiveness to plant hormone (Fig. S3). ARF5 [36], TAS14 [37], E4 [38], PR1 [39], WRKY37 [40] and D27 [41] could be induced by IAA, ABA, Ethephon, SA, MeJA and GR24 respectively, while CLAU [42], GA20ox1 [43] and $C P D$ [44] could be repressed by $6-\mathrm{BA}, \mathrm{GA}_{3}$ and $\mathrm{EBL}$ respectively. And expectedly, the expression of these genes under corresponding hormone treatment were basically in line with the reports published before, for example, TAS14 and PR1 were greatly induced 


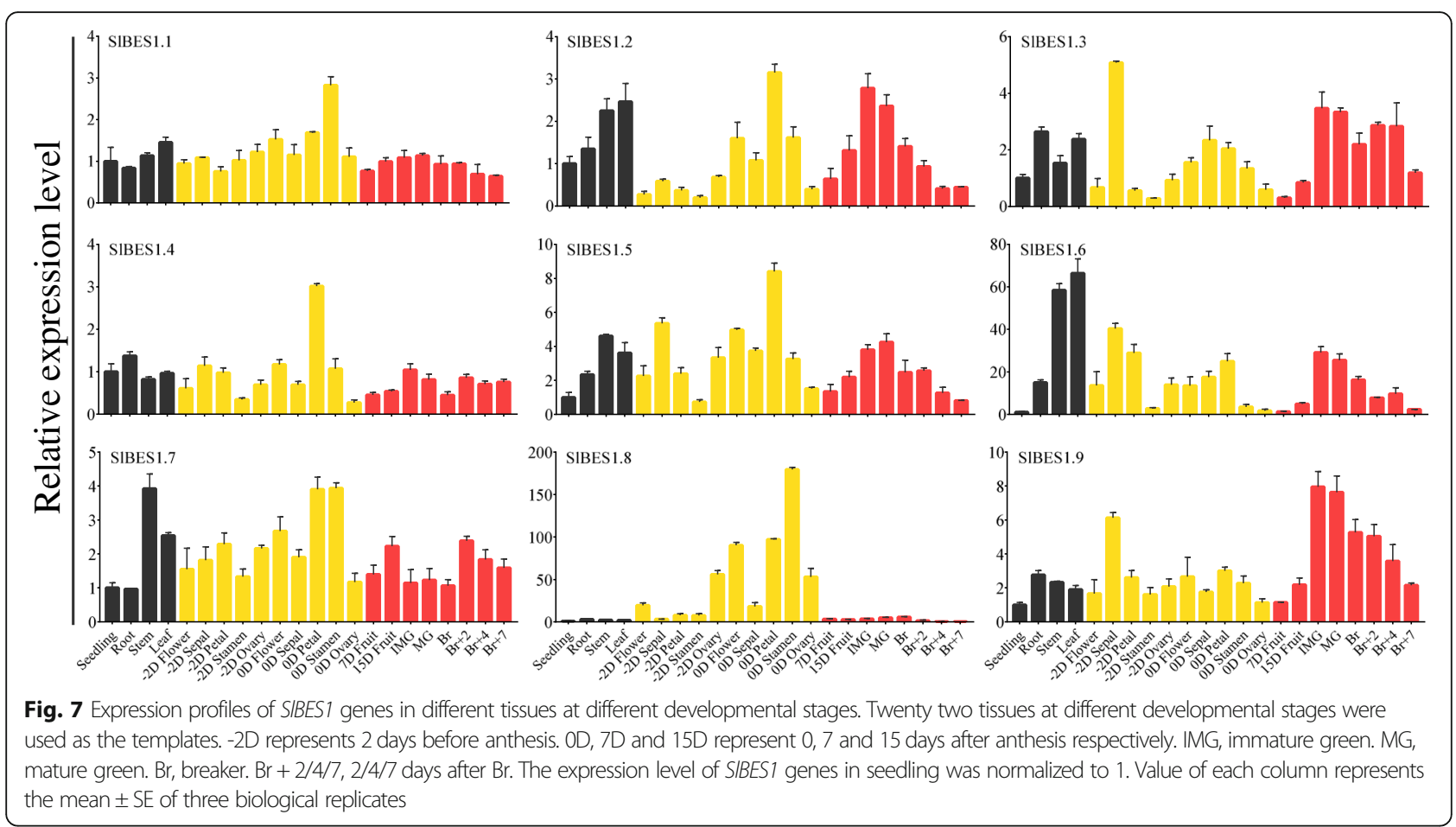

over hundreds times by $\mathrm{ABA}$ and $\mathrm{SA}$ respectively, suggesting the effective treatment of plant hormone.

The responsiveness of SlBES1 genes to these hormones was investigated by qRT-PCR (Fig. 8). The fold change of expression level more than 2 times with $p$ value lower than $0.05(\mathrm{FC}>2, p<0.05)$ was regarded as having responsiveness to the plant hormone. According to this, the responsiveness of SlBES1 genes to these nine kinds of plant hormones was summarized in Table S1 and the presence of responsiveness was marked as "Y". In general, all SIBES1 genes could response to at least one kind of plant hormone, while the responsiveness to different plant hormone was distinguishing. For example, SlBES1.6 could response to 8 kinds of plant hormones while SIBES1.9 could only response to one, i.e. GR24. On the other hand, GR24 could affect the maximum number of SlBES1 gene, up to 8 members, indicating that SlBES1 genes may have potential connection with strigolactone signaling. However, ethephon could only influence the expression of SIBES1.2. Besides, SIBES1 genes showed an identical trend in response to some plant hormones, in this case, SlBES1 genes were generally induced by IAA while repressed by GR24. On the contrary, SlBES1 genes could also be affected by some plant hormone with an opposite trend, for instance, ABA induced the expression of SIBES1.6 and SIBES1.8 while repressed SIBES1.3 and SIBES1.5. Taken together, the variational expression of SlBES1 genes under different plant hormone treatment implied that this gene family involved in multiple hormonal signals in a complicated way. The detailed role of this gene family in the crosstalk of plant hormones thus was worth to studying and may provide us the new insight in the field.

\section{Expression profiles of SIBES1 genes in response to stresses}

To further explore the potential responsiveness of SlBES1 genes to biotic and abiotic stresses, we analyzed their expression profiles to drought, osmosis, salt, oxidization, dehydration and wound stress (Fig. 9). The presence of responsiveness to these stresses was summarized in Table S2 and marked as "Y". Overall, SIBES1 gene family could be affected by multiple stresses, which principally exhibited the downregulated trend in response to all of these six stresses. This indicated that SlBES1 gene family may play the negative roles in tomato stress tolerance. In detail, four members (SlBES1.2, SlBES1.3, SlBES1.4, SIBES1.5) were hyperresponsive to all treatments analyzed here. Besides, at least four treatments can repress or induce the other five members. Notably, the strongest responsiveness of SlBES1 family genes was detected after the wound treatment. In contrast, the relative mild responsiveness was observed in salt stress. The extensive involvement of SlBES1 genes in response to these stresses implied the potential important functions of them. 


\begin{tabular}{|c|c|c|c|c|c|c|c|}
\hline & IAA & $6-\mathrm{BA}$ & & A3 & $\mathrm{ABA}$ & Ethephon & \\
\hline SIBES1.1 & & & $*$ & & * & & \\
\hline SIBES1.2 & & & & & & & * \\
\hline SlBES1.3 & * & & & & & & \\
\hline SIBES1.4 & & * & & & & & \\
\hline SlBES1.5 & $* *$ & & & & $*$ & & \\
\hline SIBES1.6 & * & * & & $*$ & $* * * *$ & & \\
\hline SIBES1.7 & & & & & & & \\
\hline SlBES1.8 & $* * * *$ & & * & $* * *$ & $* *$ & & \\
\hline SIBES1.9 & & & & & & & \\
\hline & $1 \mathrm{~h} 2 \mathrm{~h} 4 \mathrm{~h} 8 \mathrm{~h} 16 \mathrm{~h}$ & $1 \mathrm{~h} 2 \mathrm{~h} 4 \mathrm{~h} 8 \mathrm{~h} 16 \mathrm{~h}$ & $1 \mathrm{~h} 2 \mathrm{~h} 4$ & h $8 \mathrm{~h} 16 \mathrm{~h}$ & 1h $2 \mathrm{~h} 4 \mathrm{~h} 8 \mathrm{~h} 16 \mathrm{~h}$ & $1 \mathrm{~h} 2 \mathrm{~h} 4 \mathrm{~h} 8 \mathrm{~h} 16$ & \\
\hline & EBL & SA & & eJA & GR24 & & \\
\hline SlBES1.1 & & $*$ & & * & $* *$ & $\log _{2} \mathrm{FC}$ & \\
\hline SIBES1.2 & $* *$ & * & & 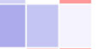 & 1 & ${ }^{3}$ & \\
\hline SIBES1.3 & & * & & * & $* *$ & 2 & \\
\hline SIBES1.4 & $*$ & 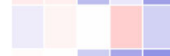 & & - & * & 1 & \\
\hline SIBES1.5 & & $* *$ & & $* *$ & $* \quad * * *$ & 0 & \\
\hline SlBES1.6 & & $*$ & & $* \quad *$ & $*$ & -1 & \\
\hline S1BES1.7 & * & & & & $* *$ & -2 & \\
\hline SIBES1.8 & * & $* * * *$ & & * & $* *$ & -3 & \\
\hline SlBES1.9 & & & & & & & \\
\hline & $1 \mathrm{~h} 2 \mathrm{~h} 4 \mathrm{~h} 8 \mathrm{~h} 16 \mathrm{~h}$ & $1 \mathrm{~h} 2 \mathrm{~h} 4 \mathrm{~h} 8 \mathrm{~h} 16 \mathrm{~h}$ & $1 \mathrm{~h} 2 \mathrm{~h} 4$ & th $8 \mathrm{~h} 16 \mathrm{~h}$ & $1 \mathrm{~h} 2 \mathrm{~h} 4 \mathrm{~h} 8 \mathrm{~h} 16 \mathrm{~h}$ & & \\
\hline $\begin{array}{l}\text { Fig. } 8 \text { Expression profiles of SIBES } \\
\text { Ethephon, EBL, SA, MeJA and GR2 } \\
\text { gibberellin, cytokinin, abscisic acid } \\
\text { seedlings treated with liquid MS/2 } \\
\text { by qRT-PCR after treated for } 1 \mathrm{~h}, 2 \\
\text { time point represents the mean o } \\
\text { respectively. * refer to significant }\end{array}$ & $\begin{array}{l}\text { S1 genes under } \\
24 \text { were selected } \\
d \text {, ethylene, brass } \\
2 \text { medium witho } \\
2 \mathrm{~h}, 4 \mathrm{~h}, 8 \mathrm{~h} \text { and } \\
\text { of three biologica } \\
\text { differences with }\end{array}$ & $\begin{array}{l}\text { mone treatment } \\
\text { treat the tomatc } \\
\text { steroid, salicylic } \\
\text { any plant hormo } \\
\text { Data were con } \\
\text { plicates. Red an } \\
0.05 \text { compared }\end{array}$ & $\begin{array}{l}\text { ne princ } \\
\text { dlings at } \\
\text { jasmoni } \\
\text { vere use } \\
\text { ed to log } \\
\text { ee colors }\end{array}$ & $\begin{array}{l}\text { ipal plant } \\
t 12 \mathrm{DPG} t \\
\text { ic acid and } \\
\mathrm{d} \text { as contr } \\
\mathrm{g}_{2} \mathrm{FC} \text { ( } \mathrm{FC} \text {, fc } \\
\text { indicate it } \\
\text { ponding }\end{array}$ & $\begin{array}{l}\text { ormones or their al } \\
\text { analyze the respo } \\
\text { trigolactone respe } \\
\text { The relative expre } \\
\text { d change) and vis } \\
\text { reased and decre } \\
\text { ock controls }\end{array}$ & $\begin{array}{l}\text { logues includin } \\
\text { iveness of SIBES } \\
\text { ively. Samples } \\
\text { sions of SIBES1 } \\
\text { lized by heat } m \\
\text { ed expression le }\end{array}$ & $\begin{array}{l}\text { ig IAA, GA } 3 \text {, 6-BA, ABA, } \\
\text { S1 genes to auxin, } \\
\text { collected from those } \\
\text { genes were detected } \\
\text { nap. Value for each } \\
\text { evels to the control }\end{array}$ \\
\hline
\end{tabular}

\section{Discussion}

$B E S 1$ transcription factors were widely present in plants. Since its definition in Arabidopsis, the genome-wide identifications of BES1 gene family had been conducted in many species, including Brassica rapa [45], Brassica napus [32], Brassica rapa ssp. pekinensis [46], Zea mays [47], Legume [31], Gossypium [29], Glycine max [48] and Malus domestica [49], while few reports focused on the functions of this gene family in tomato (Solanum lycopersicum). In our study, nine BES1 genes were identified in tomato (Table 1), in which seven members were confirmed acting as TFs by combining the investigation of subcellular localization and transactivation activity whereas another two members were not (Figs. 5, 6). According to the conserved amino acid residues analysis, SIBES1 proteins possessed the same conserved $\mathrm{N}$ terminal and serine-rich C-terminal (potentially responsible for DNA binding and phosphorylation respectively) in their BES1-type domain as observed in Arabidopsis (Fig. 1c). Besides, tomato BES1 family shared very similar gene structure with their Arabidopsis homologs, in CDS region, the exon, BES1-type domain and EARmotif showed highly identical in the number, location and length (Fig. 3b). Moreover, the conserved MEME motifs of BES1 proteins also exhibited corresponding permutation and combination with their phylogenetic relationship (Fig. 3c). These results implied the possibility that the BES1 gene family may function conserved and redundant in tomato and Arabidopsis. Indeed, AtBES1/AtBZR1 and AtBEHs exhibited functional redundancy in a certain extent, high ordered mutant of them resulted in the male sterility phenotype in Arabidopsis while single, double, triple, and quadruple mutants didn't show such a phenotype [25, 26]. Under the guidance of these results, we could assume that multiple mutant of SlBES1 genes may also influence the same biological processes.

As reported previously, BES1 proteins were phosphorylated by the core negative regulator BRASSINOSTEROIDINSENSITIVE 2 (BIN2) in the absence of BR, and the phosphorylated BES1 mainly distributed in the cytoplasm. In the presence of BR, the activity of BIN2 was inhibited, meanwhile the phosphorylated BES1 was dephosphorylated by PROTEIN PHOSPHATASE 2A (PP2A) and translocated subsequently from the cytoplasm to the nucleus $[15,50,51]$. Corresponding with this, the subcellular localization of 


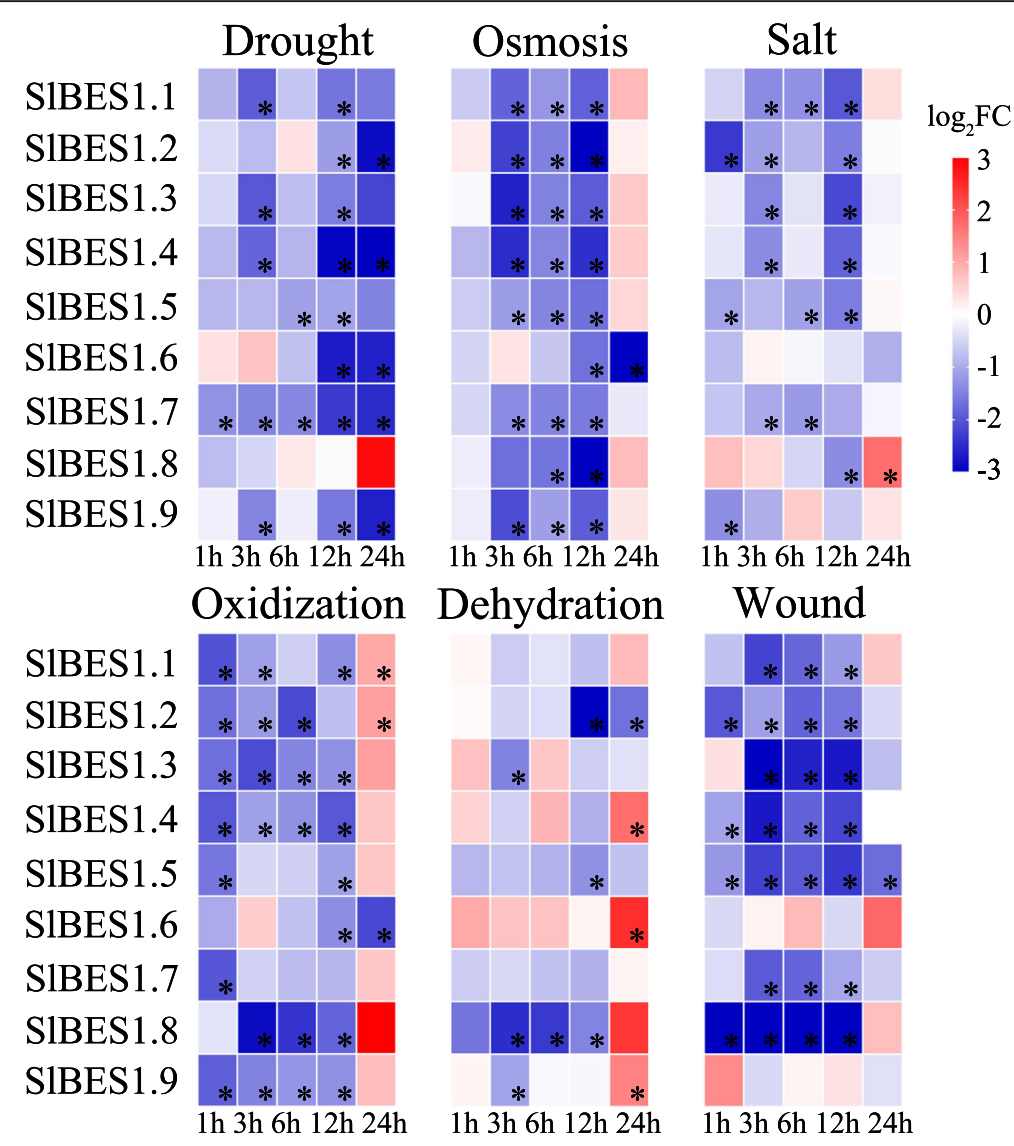

Fig. 9 Expression profiles of SIBES1 genes under stress treatments. Droughty (PEG6000), osmotic (Mannitol), oxidative (MV), salt (NaCl), dehydrated (Dehydration) and injured (Wound) stress were conducted to one-month-old tomato plants to analyze the responsiveness of SIBES1 genes. Samples collected from those tomato plants well-watered were used as control. The relative expressions of SIBESI genes were detected by qRTPCR after treated for $1 \mathrm{~h}, 3 \mathrm{~h}, 6 \mathrm{~h}, 12 \mathrm{~h}$ and $24 \mathrm{~h}$. Data were converted to $\log _{2} \mathrm{FC}$ and visualized by heat map. Value for each time point represents the mean of three biological replicates. Red and blue colors indicate increased and decreased expression levels to the control respectively. ${ }^{*}$ refer to significant differences with $p<0.05$ compared to the corresponding mock controls

AtBES1 showed the presence both in the nucleus and cytoplasm [13, 52]. Similarly, in our investigation, seven tomato BES1 members exhibited both nuclear and cytoplasmic localization (Fig. 5), which in turn suggested that tomato BES1 proteins also kept with the regulation of the phosphorylation. What's more, another two special BES1 proteins, BMY2 and BMY4, were reported to locate in the nucleus and function as TFs in Arabidopsis [27]. Different with this result, their tomato homologs SIBES1.7 and SlBES1.1 didn't localize to the nucleus (Fig. 5, S2), implying that functional differentiation of these two genes may occur. Given that four of nine Arabidopsis $\beta$-amylases located in the chloroplast [53], we investigated whether SlBES1.1 and SIBES1.7 possessed the same localization with them. While still, neither SlBES1.1-GFP nor SIBES1.7-GFP fluorescence overlapped with chlorophyll auto-fluorescence (Fig. S2), indicating the non-chloroplastic localization of SIBES1.1 and SIBES1.7. According to the size of fluorescence pigment, we supposed that these two genes may localize to endoplasmic reticulum.
More experiments need to be performed to validate the subcellular localization of SIBES1.1 and SIBES1.7.

EAR motif was a well elucidated active repression motif in plant. EAR motif-containing proteins can act as transcription factors to directly repress downstream gene transcription by histone modifications, or otherwise, act as transcription regulators to regulate the activity of transcriptional activators by binding to them, thus decreased the expression level of target genes [34, 54]. By analyzing the gene structure and amino acid sequences, we found the presence of EAR motif in all those BES1 genes annotated as TFs (Fig. 3b), which suggested the potential transcriptional repression function of these genes. Among them, however, five members (SlBES1.3, SlBES1.4, SlBES1.5, SlBES1.6 and SlBES1.9) showed the transcriptional activation activity in yeast, and only two members (SlBES1.2 and SlBES1.8) acted as transcriptional repressor (Fig. 6). In fact, it was reported that AtBZR1 played dual roles in BR homeostasis and 
signaling despite the EAR motif present in its Cterminal. In this case, AtBZR1 can not only repress BR biosynthetic genes but activate downstream BRresponsive genes by directly binding to their promoters [14]. In a recent report published by Jia et al. [55], SlBZR1 (also named SlBES1.9 in our research) functioned as transcriptional repressor, which was opposite to our result. In their investigation, the Arabidopsis leaf protoplasts were used as the material to assess the transactivation ability of SlBZR1. The expression of reporter was not significantly changed by SlBZR1 in the absence of VP16, while in the presence of VP16, the transcriptional activation activity of VP16 was significantly reduced by SIBZR1. Given the different intracellular environment between yeast and Arabidopsis leaf protoplast, we may suppose that several different proteins may influence the transactivation activity of SIBZR1 by directly interaction or competition, resulting the presence of opposite transactivation ability. Similarly, AtBES1 also possessed the EAR motif in its C-terminal and functioned both as activator and repressor [7, 15]. This phenomenon was pervasive among transcription factors, for example, Arabidopsis Yin Yang 1 (AtYY1) contained both activation and repression domains, residues 51-193 in its N-terminal showed strong repressive activity while residues $256-387$ in its C-terminal had more than 4-fold activating activity, resulting the full length of AtYY1 a weak transcriptional repression activity [56]. Thus we may assume that those SIBES1 genes exhibited transcriptional activation activity in yeast could also contain an activation domain apart from the repressive EAR motif, making them can either activate or repress downstream genes under particular circumstances.

A tomato BES1 gene, SlBES1.8, which was grouped in D in the NJ-tree (Fig. 2), had no closed homolog in Arabidopsis. Besides, it owned a simpler gene structure and only one conserved motif analyzed here (Fig. 3), implying the speciality of this gene. Subcellular localization showed that SIBES1.8 localized in the nucleus and cytoplasm (Fig. 5), combined with the transcriptional repression activity concluded by the analysis in yeast and dualluciferase assay (Fig. 6), we could regard it as a transcriptional repressor. Development- and tissue-specific expression pattern showed an exclusively relative high expression level of SIBES1.8 in floral organ (Fig. 7), which provided the possibility that SIBES1.8 may contribute to the fruit set and early development of tomato. Consistent with this assumption, SIBES1.8 could be induced by both auxin and gibberellin (Fig. 8), two important plant hormones in regulation of fruit setting and development. What's more, the auxin and gibberellin responsive cis-elements, AuxRR-core and TATC-box, were also observed in the promoter region of SIBES1.8 (Fig. 4). Taken together, we could speculate that SIBES1.8 may have important function in tomato fruit set and development, which was not reported in the function of other BES1 members.

Among all plant hormone treatments, the most obvious change in SlBES1 expression occurred when exposed to GR24, in which SlBES1 genes were significantly repressed in early treatment stages (Fig. 8). It was reported that MORE AXILLARY GROWTH LOCUS 2 (MAX2), a critical strigolactone (SL) signaling component, can interact with BES1 and its homologs and regulate AtBES1 degradation, this process was dependent on the $26 \mathrm{~S}$ proteasome and promoted by GR24 [57]. Furthermore, a putative SLs receptor, AtD14, can also enhance the degradation of AtBES1 [57]. Given that the decreased expression level of SlBES1 genes (Fig. 8), we may assume that SlBES1 proteins were under the same degradation regulation in tomato. Beyond this, however, the transcription levels of SIBES1 genes were not greatly influenced by the hormone treatments (such as ethephon) despite diacritical expression changes showed in some time points (Fig. 8). It was well elucidated that BES1 and BZR1 functioned in the dephosphorylated form, hormone treatments may not affect their mRNA levels but change their phosphorylation status. Indeed, the expression level of AtBES1 was not affected by EBL treatment, whereas appeared to be stabilized in the dephosphorylated form [13]. In this study, EBL treatment also didn't change the transcriptional levels of SIBES1 genes in the early treatment stage, while some members, such as SlBES1.6 and SlBES1.2, were induced or repressed after treated for $8 \mathrm{~h}$ (Fig. 8). Similar to EBL treatment, the expression levels of AtBES1 and AtBZR1 were also not influenced by $\mathrm{GA}_{3}$ treatment in Arabidopsis, while the dephosphorylated AtBZR1 appeared to accumulate after $\mathrm{GA}_{3}$ application [58]. In our investigation, most of SlBES1 family genes had no responsiveness to $\mathrm{GA}_{3}$ treatment, except a slight induction of SlBES1.6 and SIBES1.8 and repression of SlBES1.1 observed in some time points (Fig. 8, Table S1). AtBZR1 can interact with all DELLA proteins concluded by yeast two-hybrid. Besides, the physical protein-protein interaction of AtBZR1 with REPRESSOR OF ga1-3 (RGA), a DELLA family transcriptional regulator, was further validated by the colocalization, BiFC and co-IP assays. Moreover, AtBZR1 and RGA appeared to antagonize each other's transcriptional activity [58]. Taken together, $\mathrm{GA}_{3}$ affected the function of $B E S 1$ family primarily not in the transcriptional level but the translational level by regulating the BES1-DELLA interaction. Thus for the investigation of BES1 family mediated crosstalk with plant hormones, further studies should not only focus on the transcription 
regulation but also research the protein-protein interaction and phosphorylation status of BES1 family.

From the expression pattern in stress treatments, we can know that most of SIBES1 genes were suppressed when exposed to the stresses and thus may function in a negative way in response to these stresses (Fig. 9). Many studies had proved this assumption, for example, AtBZR1 can directly repress the expression of JUNGBRUNNEN1 (JUB1), a hydrogen peroxide-induced NAC transcription factor that functioned in promoting tolerance to various abiotic stresses $[59,60]$. Besides, a previous study confirmed that BR signaling pathway can inhibit drought response by regulating the reciprocal inhibitory mechanism between BES1 and RESPONSIVE TO DESICCATION 26 (RD26), a negative regulator of the BR pathway. Moreover, RD26 can be induced by drought and promote the expression of droughtregulated genes thus enhance drought tolerance of plant. While BES1 can repress the expression of RD26 and other drought-related genes and lead to the inhibition of drought response [61]. Hence, we speculated that knock down of SlBES1 gene family may improve the stress resistance and thus raise the yield of tomato.

Overall, this study provided valuable information for SIBES1 family, including their genomic characterization, protein functional features and expression patterns in different tissues and in response to plant hormones and stresses. The results offered important clues for functional research of SIBES1 genes and for the understanding of hormone signal crosstalk and stress resistance of tomato.

\section{Conclusions}

In this study, nine BES1 genes were identified in tomato. A systematic genome characterization was subsequently analyzed for this family, including chromosomal location, conserved amino acid residues within BES1-type domain, evolutional relationships, gene structures, conserved motifs and cis-elements. Subcellular localization and transactivation activity of SIBES1 genes were further investigated. Besides, the expression profiles of SIBES1 genes in different tissues showed potential important function in tomato fruit set and development. Moreover, the critical regulatory roles were implied by the expression patterns of SlBES1 genes in response to plant hormones and stresses. Hence, our results lay a foundation for the functional research of SIBES1 family.

\section{Methods}

\section{Plant materials and growth conditions}

Tomato plants (Solanum lycopersicum cv. Micro-Tom, a tomato dwarf cultivar obtained from Laboratory of Genomics and Biotechnology of Fruit, INRA, University of Toulouse, France) were transplanted on soil in greenhouse after germinated for 12 days on MS/2 medium.
The suitable growth conditions were set to $16 / 8 \mathrm{~h}$ light/ dark cycle, $25 / 20^{\circ} \mathrm{C}$ day/night temperature and $60 \%$ relative humidity. Tobacco plants (Nicotiana benthamiana L.) were directly planted on soil under the same growth conditions. All plants were irrigated with nutrient solution once a week. Samples analyzed in developmentand tissue-specific expression were collected from tomato seedling (12 DPG), one-month-old tomato (30 DPG) and other tomato tissues in corresponding developmental stages. Seedling, root, stem and leaf were collected from at least 8 independent healthy plants. Anthesis flower, flower at 2 days before anthesis and corresponding floral organ were collected from at least 20 independent healthy plants. Fruit samples at each stage were collected from at least 10 individual fruits. All samples were frozen immediately and mixed thoroughly after grinded. Each tissue group contained three independent biological samples and four technical repetitions for each sample were performed in qRT-PCR.

\section{Identification of $B E S 1$ genes in tomato}

To identify BES1 gene family in tomato, the AtBES1 was first obtained from The Arabidopsis Information Resource (TAIR) database (https://www.arabidopsis.org/). Full length of amino acid sequence of AtBES1 was then used to search the BES1-type domain by CD-search in NCBI (https://www. ncbi.nlm.nih.gov/cdd/?term=). The amino acid sequence of BES1-type domain was used as a BLASTP query in Solanaceae Genomics Database (http://solgenomics.net/, Tomato Genome proteins, ITAG release 4.0) with an e-value of $10^{-10}$. What's more, Phytozome database (https:// phytozome.jgi.doe.gov/pz/portal.html\#!info?alias=Org_ Slycopersicum) was also used to search BES1 gene family in tomato. The presence of BES1-type domain in candidates obtained above were further confirmed by $\mathrm{CD}$-search and SMART (http://smart.embl-heidelberg.de/). Taken together, candidates contained the BES1-type domain were regarded as $B E S 1$ genes in tomato. BES1 gene family in Arabidopsis (A. thaliana), pepper (C. annuum), soybean (G. max), rice (O. sativa) and maize ( $Z$. mays) were collected from TAIR, Solanaceae Genomics Database, Glycine max Wm82.a2.v1 (https://phytozome.jgi.doe.gov/pz/portal.html\#!linfo?alias= Org_Gmax), Rice Genome Annotation Project Database (http://rice.plantbiology.msu.edu/index.shtml) and Zea mays database (http://www.gramene.org/) respectively.

\section{Bioinformatic analyses of tomato SIBES1 genes}

The genomic loci of SIBES1 genes were collected from Solanaceae Genomics Database. Besides, the molecular weight (MW) and isoelectric point (pI) of SlBES1 genes were calculated by ProtParam tool in ExPASy Server (https://web.expasy.org/protparam/). ClustalX2.1 software [62] was used to conduct the multiple sequence alignment with full length sequences of nine SlBES1 
proteins and eight AtBES1 proteins. The alignment result was further processed by ESPript 3.0 (http://espript. ibcp.fr/ESPript/cgi-bin/ESPript.cgi) to output the picture. For the conserved amino acid residues analysis of SlBES1 genes, the BES1-type domain of each SlBES1 protein was confirmed by CD-search and subsequently visualized by WebLogo (http://weblogo.berkeley.edu/). What's more, to analyze the evolutionary relationship, full length of BES1 proteins from tomato, Arabidopsis, pepper, soybean, rice and maize were aligned by MUSCLE program in MEGA X [63] with default settings. A Neighbor-joining Tree was then constructed based on the alignment result, and the Interactive Tree Of Life (iTOLv5) online tool (https://itol.embl.de/) was finally used to polish the NJ-tree. TBtools [64] and One Step MCScanX was used for gene synteny analysis among tomato, Arabidopsis, pepper and rice, and the result was further visualized by Multiple synteny Plot. Additionally, the structure of BES1 genes was visualized by Gene Structure Display Server (GSDS 2.0) (http:// gsds.cbi.pku.edu.cn/), and the conserved amino acids motifs of BES1 proteins were explored through MEME Suite (http://meme-suite.org/tools/meme). To explored the cis-elements in the promoter region of SIBES1 genes, $2 \mathrm{~kb}$ sequences in the upstream of SIBES1 coding sequences were used to submit into PlantCARE database (http://bioinformatics.psb.ugent.be/webtools/plantcare/ $\mathrm{html} /$ ).

\section{Subcellular localization of SIBES1 proteins}

To determine the subcellular localization, full length of coding sequences without stop codon of SIBES1 genes were fused into pCXDG-GFP vector. The fusion plasmids were subsequently transformed into Agrobacterium tumefaciens (GV3101). Leaf of one-month-old tobacco was used to transiently express the fusion SIBES1-GFP proteins. The green fluorescence was observed through the laser scanning confocal microscope (Leica TCS SP8, Germany) after infected for 3 days.

\section{Transactivation activity analysis in yeast}

The open reading frames (ORFs) of SlBES1 genes were amplified and ligated into pGBKT7-GAL4BD plasmid. The fusion GAL4BD-SIBES1 constructs were further transformed into $\mathrm{Y} 2 \mathrm{H}$ Gold yeast cells. The SD/-Trp medium plates were used to cultivate the yeast transformants. The $\alpha$-galactosidase activity of the transformants was identified by X- $\alpha$-gal and the expression of AUR1-C was screened by Aureobasidin A (AbA, Clontech, USA).

\section{Dual-luciferase assay}

The ORFs of SIBES1.2 and SIBES1.8 were amplified and ligated into pEAQ-GAL4BD plasmid as the effector. Besides, VP16 was selected to constructed into pEAQ-
GAL4BD as the positive control. The double-reporter vector, pGreenII 0800-LUC, which contained the GAL4binding element $(5 \times$ GAL4 $)$ fused with the minimal TATA region of CaMV35S to drive the expression of firefly luciferase (LUC), was considered as the reporter. The renilla luciferase (REN) driven by CaMV35S was used as the internal control.

The effectors and reporter were transformed into Agrobacterium tumefaciens (GV3101) respectively, and co-infected the one-month-old tobacco leaf following the ratio of effector: reporter $=9: 1$. The activity of LUC and REN were measured after co-infected for 3 days by the Dual-Luciferase Reporter Assay System (Promega, USA). At least six biological replicates were performed for each combination. Finally, the LUC/REN ratio was calculated to assess the transcriptional activation activity of SlBES1 proteins.

\section{Hormone and stress treatments}

For hormone treatments, tomato seedlings were first germinated and grown on solid MS/2 medium. After germinated for 12 days, tomato seedlings were soaked into liquid MS/2 medium containing $20 \mu \mathrm{M}$ IAA, $10 \mu \mathrm{M}$ 6-BA, $20 \mu \mathrm{M} \mathrm{GA}, 100 \mu \mathrm{M}$ ABA, $20 \mu \mathrm{M}$ Ethephon, $0.5 \mu \mathrm{M}$ EBL, $20 \mu \mathrm{M}$ SA, $50 \mu \mathrm{M}$ MeJA and $5 \mu \mathrm{M}$ GR24 respectively and incubated in the dark at $25^{\circ} \mathrm{C}$. Samples were collected after treated for $1 \mathrm{~h}, 2 \mathrm{~h}, 4 \mathrm{~h}, 8 \mathrm{~h}$ and $16 \mathrm{~h}$ respectively. Samples collected from those seedlings soaked into MS/2 medium without any hormone at each time point were used as control. Three individual seedlings were collected for one sample, and three samples were collected for each treatment at each time point.

One-month-old tomato plants were subjected to the stress treatments. Droughty, osmotic, oxidative and salt stress treatments were carried out by soaking the tomato plants into solutions containing 20\% (m/v) PEG6000, $100 \mathrm{mM}$ mannitol, $150 \mu \mathrm{M}$ methyl viologen (MV) and $200 \mathrm{mM} \mathrm{NaCl}$ respectively followed by cultivating at standard conditions. For dehydrated stress treatment, tomato plants were removed the soil and cleaned by water, then placed on the filter papers and naturally dried at room temperature. For injured stress treatment, tomato leaves at the same position were pierced with tweezers. Tomato plants well-watered were used as control. Samples were collected after treated for $1 \mathrm{~h}, 3 \mathrm{~h}, 6 \mathrm{~h}, 12 \mathrm{~h}$ and $24 \mathrm{~h}$. Leaves at the same position of three individual plants were harvested as one sample, and three samples were collected for each treatment at each time point.

\section{RNA isolation, CDNA synthesis and quantitative real-time PCR analysis}

Total RNA was extracted with RNAprep Pure Plant Kit (Tiangen Biotech, China) according to the manufacturer's instructions. The integrity of total RNA was detected by 
agarose gel electrophoresis and the concentration was measured by NanoDrop 1000 (Thermo, USA). The first strand cDNA was synthesized by PrimeScript ${ }^{\mathrm{Ts}} \mathrm{RT}$ reagent Kit with gDNA Eraser (Perfect Real Time) (Takara, Japan) with $2 \mu \mathrm{g}$ total RNA for each $40 \mu \mathrm{L}$ reaction. The cDNA products were diluted to 5 -fold with deionized water before use. TB Green ${ }^{\odot}$ Premix Ex Taq ${ }^{\mathrm{Tm}}$ II (Tli RNaseH Plus) (Takara, Japan) was used to conduct qRT-PCR on the CFX96 Touch ${ }^{\text {тм }}$ Real-Time PCR Detection System (BIORAD, USA). Two microliter diluted cDNA was used in each reaction, other reaction components and conditions were performed following the manufacturer's instructions. The relative expression was calculated by the $2^{-\Delta \Delta C t}$ method and visualized as heatmaps by TBtools.

\begin{abstract}
Abbreviations
AA: Amino acid; AbA: Aureobasidin A; BES1: BRI1-EMS-SUPPRESSOR 1; BZR1: BRASSINAZOLE-RESISTANT 1; CDS: Coding sequence; ChIPchip: Chromatin immunoprecipitation-microarray; EAR: Ethylene-responsive element binding factor-associated amphiphilic repression; GFP: Green fluorescent protein; MW: Molecular Weight; ORFs: Open reading frames; pl: Theoretical Isoelectric Point; qRT-PCR: Quantitative real-time polymerase chain reaction; TFs: Transcription factors; UTR: Untranslated region
\end{abstract}

\section{Supplementary Information}

The online version contains supplementary material available at https://doi. org/10.1186/s12870-021-02933-7.

Additional file 1: Figure S1. The detailed sequence logos of those 10 conserved motifs in MEME analysis. Figure S2. Subcellular localization analysis of SIBES1.1 and SIBES1.7. Figure S3. Relative expression of the reference genes under corresponding hormone treatments. Table S1. Summary of the responsiveness of SIBES1 family to hormone treatments. Table S2. Summary of the responsiveness of SIBES1 family to stress treatments. Table S3. Primers used in this study.

\section{Acknowledgements}

We would like to thank Analytical and Testing Center of Chongqing University for providing laser scanning confocal microscope analysis.

\section{Authors' contributions}

Z. L. and Y. L. designed the research; D. S., W. X., L. W. and W. L. performed the experiments and conducted the bioinformatics analysis; D. S. wrote the manuscript and Y. L. and Y. S. revised the paper. All authors had read and approved the final manuscript.

\section{Funding}

This work was supported by the National Key Research and Development Program (2016YFD0400101), and the National Natural Science Foundation of China (No. 31772370, 31972470, 32002100). The funding bodies played no role in the design of the study and collection, analysis, and interpretation of data and in writing the manuscript.

\section{Availability of data and materials}

The sequence information of tomato and Arabidopsis BES1 family genes were collected from Solanaceae Genomics Network (SGN, SL4.0, https:// solgenomics.net/) and The Arabidopsis Information Resource (https://www. arabidopsis.org/) respectively. The amino acid sequences of BES1 proteins in pepper, soybean, rice and maize were collected from SGN, soybean genome database (Wm82.a2.v1, http://phytozome.jgi.doe.gov/pz/portal.html), Rice Genome Annotation Project Database (http://rice.plantbiology.msu.edu/ index.shtml) and Zea mays database (http://www.gramene.org/) respectively. Cis-elements were obtained from PlantCARE database (http://bioinformatics. psb.ugent.be/webtools/plantcare/html/). The BES1 family expression data were generated by qRT-PCR and were available from the corresponding authors when needed. All other data supporting the results are included within the article and its Additional files.

\section{Declarations}

Ethics approval and consent to participate

Plant materials (Solanum lycopersicum cv. Micro-Tom) used in this article were obtained from the Laboratory of Genomics and Biotechnology of Fruit, INRA, University of Toulouse, Toulouse, France. All plant materials were provided free of charge and maintained in accordance with the international guidelines. This article did not contain any studies with human participants or animals and did not involve any endangered or protected species.

Consent for publication

Not applicable.

\section{Competing interests}

The authors declare that they have no competing interests.

\section{Author details}

${ }^{1}$ Key Laboratory of Plant Hormones and Development Regulation of Chongqing, School of Life Sciences, Chongqing University, Chongqing 401331, China. ${ }^{2}$ Center of Plant Functional Genomics, Institute of Advanced Interdisciplinary Studies, Chongqing University, Chongqing 401331, China.

Received: 24 December 2020 Accepted: 17 March 2021

Published online: 30 March 2021

\section{References}

1. Pauwels L, Barbero GF, Geerinck J, Tilleman S, Grunewald W, Pérez AC, et al. NINJA connects the co-repressor TOPLESS to jasmonate signalling. Nature. 2010;464(7289):788-91. https://doi.org/10.1038/nature08854.

2. Ku YS, Sintaha M, Cheung MY, Lam HM. Plant hormone signaling Crosstalks between biotic and abiotic stress responses. Int J Mol Sci. 2018;19(10):3206. https://doi.org/10.3390/ijms19103206.

3. Mitchell JW, Mandava N, Worley JF, Plimmer JR, Smith MV. Brassins-a new family of plant hormones from rape pollen. Nature. 1970;225(5237):1065-6. https://doi.org/10.1038/2251065a0.

4. Grove MD, Spencer GF, Rohwedder WK, Mandava N, Worley JF, Warthen JD, et al. Brassinolide, a plant growth-promoting steroid isolated from Brassica napus pollen. Nature. 1979;281(5728):216-7. https://doi.org/10.1038/281216a0.

5. Wang ZY, Bai MY, Oh E, Zhu JY. Brassinosteroid signaling network and regulation of photomorphogenesis. Annu Rev Genet. 2012;46(1):701-24. https://doi.org/10.1146/annurev-genet-102209-163450.

6. Planas-Riverola A, Gupta A, Betegón-Putze I, Bosch N, Ibañes M, CañoDelgado Al. Brassinosteroid signaling in plant development and adaptation to stress. Development. 2019;146(5):dev151894. https://doi.org/10.1242/ dev.151894.

7. Li QF, Lu J, Yu JW, Zhang CQ, He JX, Liu QQ. The brassinosteroid-regulated transcription factors BZR1/BES1 function as a coordinator in multisignalregulated plant growth. Biochim Biophys Acta Gene Regul Mech. 2018; 1861(6):561-71. https://doi.org/10.1016/j.bbagrm.2018.04.003.

8. Li J, Chory J. A putative leucine-rich repeat receptor kinase involved in brassinosteroid signal transduction. Cell. 1997;90(5):929-38. https://doi.org/1 0.1016/S0092-8674(00)80357-8.

9. Wang ZY, Seto H, Fujioka S, Yoshida S, Chory J. BRI1 is a critical component of a plasma-membrane receptor for plant steroids. Nature. 2001;410(6826): 380-3. https://doi.org/10.1038/35066597.

10. Kim TW, Guan S, Burlingame AL, Wang ZY. The CDG1 kinase mediates brassinosteroid signal transduction from BRI1 receptor kinase to BSU1 phosphatase and GSK3-like kinase BIN2. Mol Cell. 2011;43(4):561-71. https:// doi.org/10.1016/j.molcel.2011.05.037.

11. He JX, Gendron JM, Yang Y, Li J, Wang ZY. The GSK3-like kinase BIN2 phosphorylates and destabilizes BZR1, a positive regulator of the brassinosteroid signaling pathway in Arabidopsis. Proc Natl Acad Sci U S A. 2002;99(15):10185-90. https://doi.org/10.1073/pnas.152342599.

12. Wang ZY, Nakano T, Gendron J, He J, Chen M, Vafeados D, et al. Nuclearlocalized BZR1 mediates brassinosteroid-induced growth and feedback suppression of brassinosteroid biosynthesis. Dev Cell. 2002;2(4):505-13. https://doi.org/10.1016/\$1534-5807(02)00153-3. 
13. Yin Y, Wang ZY, Mora-Garcia S, Li J, Yoshida S, Asami T, et al. BES1 accumulates in the nucleus in response to brassinosteroids to regulate gene expression and promote stem elongation. Cell. 2002;109(2):181-91. https://doi.org/10.1016/S0092-8674(02)00721-3.

14. He JX, Gendron JM, Sun Y, Gampala SS, Gendron N, Sun CQ, et al. BZR1 is a transcriptional repressor with dual roles in brassinosteroid homeostasis and growth responses. Science. 2005;307(5715):1634-8. https://doi.org/10.1126/ science. 1107580

15. Yin Y, Vafeados D, Tao Y, Yoshida S, Asami T, Chory J. A new class of transcription factors mediates brassinosteroid-regulated gene expression in Arabidopsis. Cell. 2005;120(2):249-59. https://doi.org/10.1016/j.cell.2004.11.044

16. Xie L, Yang C, Wang X. Brassinosteroids can regulate cellulose biosynthesis by controlling the expression of CESA genes in Arabidopsis. J Exp Bot. 2011; 62(13):4495-506. https://doi.org/10.1093/jxb/err164.

17. Wang X, Zhang J, Yuan M, Ehrhardt DW, Wang Z, Mao T. Arabidopsis microtubule destabilizing protein40 is involved in brassinosteroid regulation of hypocotyl elongation. Plant Cell. 2012;24(10):4012-25. https://doi.org/1 $0.1105 /$ tpc.112.103838.

18. Kim Y, Song JH, Park SU, Jeong YS, Kim SH. Brassinosteroid-induced transcriptional repression and Dephosphorylation-dependent protein degradation negatively regulate BIN2-interacting AIF2 (a BR signalingnegative regulator) bHLH transcription factor. Plant Cell Physiol. 2017;58(2): 227-39. https://doi.org/10.1093/pcp/pcw223.

19. Zhang LY, Bai MY, Wu J, Zhu JY, Wang H, Zhang Z, et al. Antagonistic HLH/ bHLH transcription factors mediate brassinosteroid regulation of cell elongation and plant development in rice and Arabidopsis. Plant Cell. 2009; 21(12):3767-80. https://doi.org/10.1105/tpc.109.070441.

20. Li L, Yu X, Thompson A, Guo M, Yoshida S, Asami T, et al. Arabidopsis MYB30 is a direct target of BES1 and cooperates with BES1 to regulate brassinosteroid-induced gene expression. Plant J. 2009;58(2):275-86. https:// doi.org/10.1111/j.1365-313X.2008.03778.X.

21. Ye H, Li L, Guo H, Yin Y. MYBL2 is a substrate of GSK3-like kinase BIN2 and acts as a corepressor of BES1 in brassinosteroid signaling pathway in Arabidopsis. Proc Natl Acad Sci U S A. 2012;109(49):20142-7. https://doi. org/10.1073/pnas.1205232109.

22. Zhang T, Xu P, Wang W, Wang S, Caruana JC, Yang HQ, et al. Arabidopsis Gprotein $\beta$ Subunit AGB1 interacts with BES1 to regulate Brassinosteroid signaling and cell elongation. Front Plant Sci. 2017:8:2225.

23. Sun Y, Fan XY, Cao DM, Tang W, He K, Zhu JY, et al. Integration of brassinosteroid signal transduction with the transcription network for plant growth regulation in Arabidopsis. Dev Cell. 2010;19(5):765-77. https://doi. org/10.1016/j.devcel.2010.10.010.

24. Yu X, Li L, Zola J, Aluru M, Ye H, Foudree A, et al. A brassinosteroid transcriptional network revealed by genome-wide identification of BESI target genes in Arabidopsis thaliana. Plant J. 2011;65(4):634-46. https://doi. org/10.1111/j.1365-313X.2010.04449.x.

25. Chen $W$, LV M, Wang Y, Wang PA, Cui $Y$, Li M, et al. BES1 is activated by EMS1-TPD1-SERK1/2-mediated signaling to control tapetum development in Arabidopsis thaliana. Nat Commun. 2019;10(1):4164. https://doi.org/10.1038/ s41467-019-12118-4.

26. Chen LG, Gao Z, Zhao Z, Liu X, Li Y, Zhang Y, et al. BZR1 family transcription factors function redundantly and indispensably in BR signaling but exhibit BRI1-independent function in regulating anther development in Arabidopsis. Mol Plant. 2019;12(10):1408-15. https://doi.org/10.1016/j.molp.2019.06.006.

27. Reinhold H, Soyk S, Simková K, Hostettler C, Marafino J, Mainiero S, et al. $\beta$ Amylase-like proteins function as transcription factors in Arabidopsis, controlling shoot growth and development. Plant Cell. 2011;23(4):1391-403. https://doi.org/10.1105/tpc.110.081950.

28. Giovannoni J, Nguyen C, Ampofo B, Zhong S, Fei Z. The Epigenome and transcriptional dynamics of fruit ripening. Annu Rev Plant Biol. 2017;68(1): 61-84. https://doi.org/10.1146/annurev-arplant-042916-040906.

29. Liu Z, Qanmber G, Lu L, Qin W, Liu J, Li J, et al. Genome-wide analysis of BES1 genes in Gossypium revealed their evolutionary conserved roles in brassinosteroid signaling. Sci China Life Sci. 2018;61(12):1566-82. https://doi. org/10.1007/s11427-018-9412-x.

30. Li J, Nam KH. Regulation of brassinosteroid signaling by a GSK3/SHAGGYlike kinase. Science. 2002;295(5558):1299-301. https://doi.org/10.1126/ science.1065769.

31. Li Y, He L, Li J, Chen J, Liu C. Genome-wide identification, characterization, and expression profiling of the legume BZR transcription factor gene family. Front Plant Sci. 2018;9:1332. https://doi.org/10.3389/fpls.2018.01332.
32. Song X, Ma X, Li C, Hu J, Yang Q, Wang T, et al. Comprehensive analyses of the BES1 gene family in Brassica napus and examination of their evolutionary pattern in representative species. BMC Genomics. 2018;19(1): 346. https://doi.org/10.1186/s12864-018-4744-4.

33. Xu G, Guo C, Shan $H$, Kong H. Divergence of duplicate genes in exon-intron structure. Proc Natl Acad Sci U S A. 2012;109(4):1187-92. https://doi.org/10.1 073/pnas.1109047109

34. Ohta M, Matsui K, Hiratsu K, Shinshi H, Ohme-Takagi M. Repression domains of class II ERF transcriptional repressors share an essential motif for active repression. Plant Cell. 2001;13(8):1959-68. https://doi.org/10.1105/TPC.010127.

35. Sadowski I, Ma J, Triezenberg S, Ptashne M. GAL4-VP16 is an unusually potent transcriptional activator. Nature. 1988;335(6190):563-4. https://doi. org/10.1038/335563a0.

36. Zouine M, Fu Y, Chateigner-Boutin AL, Mila I, Frasse P, Wang H, et al. Characterization of the tomato ARF gene family uncovers a multi-levels post-transcriptional regulation including alternative splicing. PLoS One. 2014;9(1):e84203. https://doi.org/10.1371/journal.pone.0084203.

37. Pye MF, Dye SM, Resende RS, MacDonald JD, Bostock RM. Abscisic acid as a dominant signal in tomato during salt stress predisposition to Phytophthora root and crown rot. Front Plant Sci. 2018;9:525. https://doi.org/10.3389/fpls.2 018.00525.

38. Xu R, Goldman S, Coupe S, Deikman J. Ethylene control of E4 transcription during tomato fruit ripening involves two cooperative cis elements. Plant Mol Biol. 1996;31(6):1117-27. https://doi.org/10.1007/BF00040829.

39. Li T, Huang Y, Xu ZS, Wang F, Xiong AS. Salicylic acid-induced differential resistance to the tomato yellow leaf curl virus among resistant and susceptible tomato cultivars. BMC Plant Biol. 2019;19(1):173. https://doi.org/1 0.1186/s12870-019-1784-0.

40. Wang Z, Liu L, Su H, Guo L, Zhang J, Li Y, et al. Jasmonate and aluminum crosstalk in tomato: identification and expression analysis of WRKYs and ALMTs during JA/Al-regulated root growth. Plant Physiol Biochem. 2020;154 409-18. https://doi.org/10.1016/j.plaphy.2020.06.026.

41. Guillotin B, Etemadi M, Audran C, Bouzayen M, Bécard G, Combier JP. SIIAA27 regulates strigolactone biosynthesis and mycorrhization in tomato (var. MicroTom). New Phytol. 2017;213(3):1124-32. https://doi.org/10.1111/ nph.14246.

42. Bar M, Israeli A, Levy M, Ben Gera H, Jiménez-Gómez JM, Kouril S, et al. CLAUSA is a MYB transcription factor that promotes leaf differentiation by attenuating Cytokinin signaling. Plant Cell. 2016;28(7):1602-15. https://doi. org/10.1105/tpc.16.00211.

43. Livne S, Lor VS, Nir I, Eliaz N, Aharoni A, Olszewski NE, et al. Uncovering DELLA-independent gibberellin responses by characterizing new tomato procera mutants. Plant Cell. 2015;27(6):1579-94. https://doi.org/10.1105/ tpc.114.132795.

44. Yin $Y$, Qin $K$, Song $X$, Zhang Q, Zhou $Y$, Xia $X$, et al. BZR1 transcription factor regulates heat stress tolerance through FERONIA receptor-like kinasemediated reactive oxygen species signaling in tomato. Plant Cell Physiol. 2018;59(11):2239-54.

45. Saha G, Park Jl, Jung HJ, Ahmed NU, Kayum MA, Kang JG, et al. Molecular characterization of BZR transcription factor family and abiotic stress induced expression profiling in Brassica rapa. Plant Physiol Biochem. 2015;92:92-104. https://doi.org/10.1016/j.plaphy.2015.04.013.

46. Wu P, Song X, Wang Z, Duan W, Hu R, Wang W, et al. Genome-wide analysis of the BES1 transcription factor family in Chinese cabbage (Brassica rapa ssp. pekinensis). Plant Growth Regul. 2016;80(3):291-301. https://doi. org/10.1007/s10725-016-0166-y.

47. Manoli A, Trevisan S, Quaggiotti S, Varotto S. Identification and characterization of the BZR transcription factor family and its expression in response to abiotic stresses in Zea mays L. Plant Growth Regul. 2018;84(3): 423-36. https://doi.org/10.1007/s10725-017-0350-8.

48. Li Q, Guo L, Wang H, Zhang Y, Fan C, Shen Y. In silico genome-wide identification and comprehensive characterization of the BES1 gene family in soybean. Heliyon. 2019;5(6):e01868. https://doi.org/10.1016/j.heliyon.2019. e01868.

49. Cao X, Khaliq A, Lu S, Xie M, Ma Z, Mao J, et al. Genome-wide identification and characterization of the BES1 gene family in apple (Malus domestica). Plant Biol (Stuttg). 2020;22(4):723-33. https://doi.org/10.1111/plb.13109.

50. Kim TW, Guan S, Sun Y, Deng Z, Tang W, Shang JX, et al. Brassinosteroid signal transduction from cell-surface receptor kinases to nuclear transcription factors. Nat Cell Biol. 2009;11(10):1254-60. https://doi.org/10.1 038/ncb1970. 
51. Tang W, Yuan M, Wang R, Yang Y, Wang C, Oses-Prieto JA, et al. PP2A activates brassinosteroid-responsive gene expression and plant growth by dephosphorylating BZR1. Nat Cell Biol. 2011;13(2):124-31. https://doi.org/1 $0.1038 /$ ncb2151.

52. Jiang J, Zhang C, Wang X. A recently evolved isoform of the transcription factor BES1 promotes brassinosteroid signaling and development in Arabidopsis thaliana. Plant Cell. 2015;27(2):361-74. https://doi.org/10.1105/ tpc.114.133678.

53. Fulton DC, Stettler M, Mettler T, Vaughan CK, Li J, Francisco P, et al. BetaAMYLASE4, a noncatalytic protein required for starch breakdown, acts upstream of three active beta-amylases in Arabidopsis chloroplasts. Plant Cell. 2008;20(4):1040-58. https://doi.org/10.1105/tpc.107.056507.

54. Yang J, Liu Y, Yan H, Tian T, You Q, Zhang L, et al. PlantEAR: functional analysis platform for plant EAR motif-containing proteins. Front Genet. 2018; 9:590. https://doi.org/10.3389/fgene.2018.00590.

55. Jia C, Zhao S, Bao T, Zhao P, Peng K, Guo Q, et al. Tomato BZR/BES transcription factor SIBZR1 positively regulates BR signaling and salt stress tolerance in tomato and Arabidopsis. Plant Sci. 2021;302:1 10719. https://doi. org/10.1016/j.plantsci.2020.110719.

56. Li T, Wu XY, Li H, Song JH, Liu JY. A dual-function transcription factor, AtYY1, is a novel negative regulator of the Arabidopsis ABA response network. Mol Plant. 2016;9(5):650-61. https:/doi.org/10.1016/.jmolp.2016.02.010,

57. Wang Y, Sun S, Zhu W, Jia K, Yang H, Wang X. Strigolactone/MAX2-induced degradation of brassinosteroid transcriptional effector BES1 regulates shoot branching. Dev Cell. 2013;27(6):681-8. https://doi.org/10.1016/j.devcel.2 013.11.010.

58. Li QF, Wang C, Jiang L, Li S, Sun SS, He JX. An interaction between BZR1 and DELLAs mediates direct signaling crosstalk between brassinosteroids and gibberellins in Arabidopsis. Sci Signal. 2012;5(244):ra72.

59. Wu A, Allu AD, Garapati P, Siddiqui H, Dortay H, Zanor Ml, et al. JUNGBRUNNEN1, a reactive oxygen species-responsive NAC transcription factor, regulates longevity in Arabidopsis. Plant Cell. 2012;24(2):482-506. https://doi.org/10.1105/tpc.111.090894.

60. Shahnejat-Bushehri S, Tarkowska D, Sakuraba Y, Balazadeh S. Arabidopsis NAC transcription factor JUB1 regulates GA/BR metabolism and signalling. Nat Plants. 2016;2(3):16013. https://doi.org/10.1038/nplants.2016.13.

61. Ye H, Liu S, Tang B, Chen J, Xie Z, Nolan TM, et al. RD26 mediates crosstalk between drought and brassinosteroid signalling pathways. Nat Commun. 2017:8(1):14573. https://doi.org/10.1038/ncomms14573.

62. Larkin MA, Blackshields G, Brown NP, Chenna R, McGettigan PA, McWilliam $H$, et al. Clustal W and Clustal X version 2.0. Bioinformatics. 2007;23(21): 2947-8. https://doi.org/10.1093/bioinformatics/btm404.

63. Kumar S, Stecher G, Li M, Knyaz C, Tamura K. MEGA X: molecular evolutionary genetics analysis across computing platforms. Mol Biol Evol. 2018;35(6):1547-9. https://doi.org/10.1093/molbev/msy096.

64. Chen C, Chen H, Zhang Y, Thomas HR, Frank MH, He Y, et al. TBtools: an integrative toolkit developed for interactive analyses of big biological data. Mol Plant. 2020;13(8):1194-202. https://doi.org/10.1016/j.molp.2020.06.009.

\section{Publisher's Note}

Springer Nature remains neutral with regard to jurisdictional claims in published maps and institutional affiliations.

Ready to submit your research? Choose BMC and benefit from:
- fast, convenient online submission
- thorough peer review by experienced researchers in your field
- rapid publication on acceptance
- support for research data, including large and complex data types
- gold Open Access which fosters wider collaboration and increased citations
- maximum visibility for your research: over 100M website views per year
At BMC, research is always in progress.
Learn more biomedcentral.com/submissions

